\title{
MAGNETIZATION MEASUREMENTS FOR DILUTE ALLOYS OF Ni
}

\author{
D. Hunter $\left({ }^{1}\right)$, A. S. Arrott $\left({ }^{1}\right)$, R. I. Grynszpan $\left({ }^{2}\right)^{1}$, P. Dassonwalle $\left({ }^{2}\right)$ and P. Langlois $\left({ }^{2}\right)$ \\ $\left({ }^{1}\right)$ Physics Department, Simon Fraser University Burnaby, British Columbia, Canada V5A 1 S6 \\ (2) Centre d'Etudes de Chimie Metallurgique, Vitry, France
}

Abstract. - Precision measurements at $290.45 \mathrm{~K}$ and $293.55 \mathrm{~K}$ are used to estimate the spontaneous magnetization at $0 \mathrm{~K}$ for dilute alloys of $\mathrm{Ni}$ with $\mathrm{Ti}, \mathrm{V}, \mathrm{Cr}, \mathrm{Mn}, \mathrm{Fe}, \mathrm{Co}, \mathrm{Cu}, \mathrm{Zr}, \mathrm{Nb}, \mathrm{Mo}, \mathrm{Pd}, \mathrm{Hf}, \mathrm{Ta}, \mathrm{W}, \mathrm{Pt}$ and Au. The results are compared with the calculations of Stefanou et al. (Phys. Rev. B 35 (1987) 6911) for single impurity atoms in Ni.

In a series of papers, Stefanou et al. [1], Zeller [2] and Blügel et al. [3] have used state-of-the-art methods to understand the the role of screening by $\mathrm{d}$ states and sp states in determining the moment on solute atoms and the perturbation of the moments on the surrounding Ni atoms. Their numbers for the net spin density associated with these sites are converted to magnetic moments by assuming something about the $g$-factors, which they do not calculate. The simplest assumption is that the solute has its $g$-factor, to be deduced from whatever experiments shed light on the behavior of the solute in various metallic environments, while all the $\mathrm{Ni}$ atoms have the $g$-factor of pure $\mathrm{Ni}$, which is determined by Ferromagnetic Resonance (FMR). This yields the following expression for $y(0, c)$, the relative change in magnetic moment with atomic concentration:

$$
\begin{aligned}
y(0, c) & \equiv \frac{\sigma(0, c)-\sigma(0,0)}{\sigma(0,0)}= \\
= & \frac{\frac{w}{0.583}-\left(\frac{g_{\mathrm{Ni}}-g_{\mathrm{s}}}{g_{\mathrm{Ni}}}\right) \frac{p}{0.583}+\frac{A_{\mathrm{Ni}}-A_{\mathrm{s}}}{A_{\mathrm{Ni}}}}{1-c \frac{A_{\mathrm{Ni}}-A_{\mathrm{s}}}{A_{\mathrm{Ni}}}}
\end{aligned}
$$

where the $\sigma(0, c)$ is the moment per unit mass at $0 \mathrm{~K}$ for the alloy of concentration $c$ and atomic weight $A_{8}$, $w$ is the total excess moment due to the solute atom, $p$ is the local moment of the solute atom, and 0.583 is a calculated number of Bohr magnetons using $g_{\mathrm{e}}$, the $g$ factor of the electron. The numbers for $w$ and $p$ are given as $\Delta M$ (or $\Delta M^{\mathrm{cl}}$ ) and $M^{\mathrm{imp}}$ in table I of Stefanou et al. [1] e.g. $w=-5.319$ (or -5.300 ) and $p=-1.700$ for $\mathrm{Cr}$. The solute atom carries a local

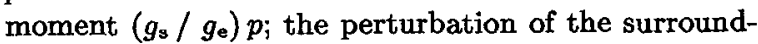
ing nickel atoms gives an additional moment per solute atom $\left(g_{\mathrm{Ni}} / g_{\mathrm{e}}\right)(w-p+0.583)$.

Given a set of properly prepared spheres of dilute $\mathrm{Ni}$ alloys and a magnetometer that measures at $4.2 \mathrm{~K}$, it would be a simple matter to obtain the experimental values of $y(0, c)$. The data are sparse. It happens that a series of dilute alloys starting from high purity nickel were made for the purpose of studying muon spin rotation and positron annihilation [4]. To extract the hyperfine fields from the muon rotation frequencies it is necessary to subtract the Lorentz field. As the muon measurements were made over a range of ambient temperatures, the magnetization was measured at two temperatures for interpolation. The result is data for the magnetization of a series of dilute $\mathrm{Ni}$ alloys at $T=290.45 \mathrm{~K}$ and $T_{1}=293.55 \mathrm{~K}$ which have suffcient precision that we attempt to deduce $y(0, c)$ from measurements of $y(T, c)$ and $\Delta_{2}\left(T, T_{1}, c\right)$ where

$$
y(T, c) \equiv \frac{\sigma(T, c)-\sigma(T, 0)}{\sigma(T, 0)}
$$

and

$$
\begin{aligned}
& \Delta_{2}\left(T, T_{1}, c\right) \equiv \\
& \quad \equiv \frac{\left\{\sigma(T, c)-\sigma\left(T_{1}, c\right)\right\}-\left\{\sigma(T, 0)-\sigma\left(T_{1}, 0\right)\right\}}{\sigma(T, 0)-\sigma\left(T_{1}, 0\right)} .
\end{aligned}
$$

If pure $\mathrm{Ni}$ and the alloys scale with temperature according to

$$
\frac{\sigma(T, c)-\sigma(0, c)}{\sigma(0, c)}=-A\left(\frac{T}{T_{c}(c)}\right)^{r},
$$

with the same $r, y(0, c)$ can be calculated using

$$
\begin{aligned}
y(0, c)=y(T, c) & \frac{\sigma(T, 0)}{\sigma(0,0)}+ \\
& +\Delta_{2}\left(T, T_{1}, c\right)\left\{1-\frac{\sigma(T, 0)}{\sigma(0,0)}\right\} .
\end{aligned}
$$

The ratio $\sigma(290.45 K, 0) / \sigma(0,0)=0.9432$ is accurately known from the work of Pauthenet [5]. That these are dilute alloys, with $0.0011<c<0.015$, should give some confidence in the assumption of constant $r$. But, it may not be a good assumption when the solute is Mn. Such an effect was found by Jaccarino et al. [6] for dilute alloys of $\mathrm{Mn}$ in $\mathrm{Fe}$.

As explained previously [4] these samples are cylinders (not spheres) and they have holes in them because of the way they were made. The method of magnetization measurement produces numbers which are reproducible to \pm 1 part per 100,000 . Each alloy sample is compared with a similarly shaped Ni standard, made by the same procedures. The measured fractional change of $\mathrm{Ni}$ moment from $290.45 \mathrm{~K}$ to $293.55 \mathrm{~K}$ agrees with that of Pauthenet [5] to a part in 400 .

As there is reason to be skeptical about extrapo-

\footnotetext{
${ }^{1}$ Present address: Laboratoire Microstructure et Mécanique des Matériaux, Ecole nationale Supérieure d'Arts et Métiers, 151 boulevard de l'Hopital, 75640 Paris Cedex 13, France.
} 
lating to $0 \mathrm{~K}$ on the basis of measurements made at $290.45 \mathrm{~K}$ and $293.55 \mathrm{~K}$, the results are shown in fig-
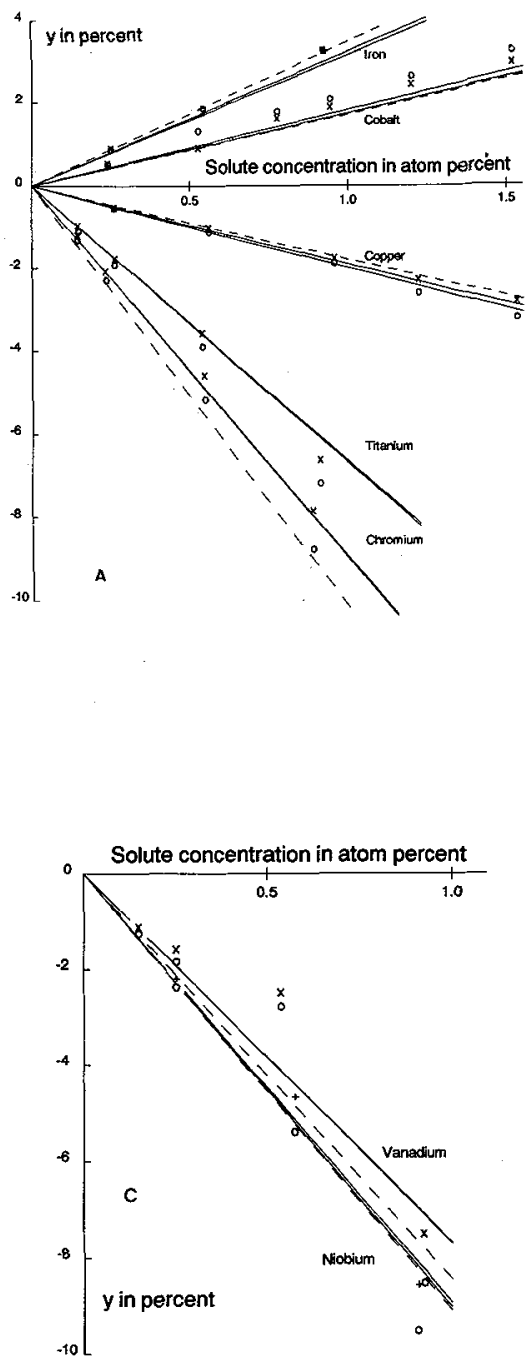

Fig. 1. - (a-d) Dilute alloys in Ni. The relative magnetic moment per unit mass $y(0, c)=[\sigma(0, c)-\sigma(0,0)] / \sigma(0,0)$ obtained starting from theory \{solid lines\} or from measurements $(\chi$ or + ) using equation (4). the points $(O)$ are computed setting $\Delta_{2}=0$. The theory lines are calculated using $g_{\mathrm{s}}=g_{\mathrm{Ni}}$ for two values given by Stefanou et al. [1]. The broken lines are calculations using the friedel rule $\{-\Delta Z$ or $10+\Delta Z\}$. (a) Some elements in the First transition series. Experiment and theory both match the Friedel rule for Titanium. The theory seems to be slightly low for $\mathrm{Cu}, \mathrm{Co}$ and $\mathrm{Fe}$. (b) The theory for Pd predicts a moment on the $\mathrm{Pd}$ atom less than that of $\mathrm{Ni}$, where as the experiment

[1] Stefanou, N., Oswald, A.., Zeller, R. and Dederichs, P. H., Phys. Rev. B 35 (1987) 6911-6922.

[2] Zeller, R., J. Phys. F. 17 (1987) 2123-2137.

[3] Blügel, S., Akai, H., Zeller, R. and Dederichs, P. H., Phys. Rev. B 35 (1987) 3271.

[4] Hunter, D., Arrott, A. S., Grynszpan, R. I., Das- ure 1 as pairs with $\left(\mathrm{x}\right.$ or + ) and without $(0)$ the $\Delta_{2}$ term in equation (4).
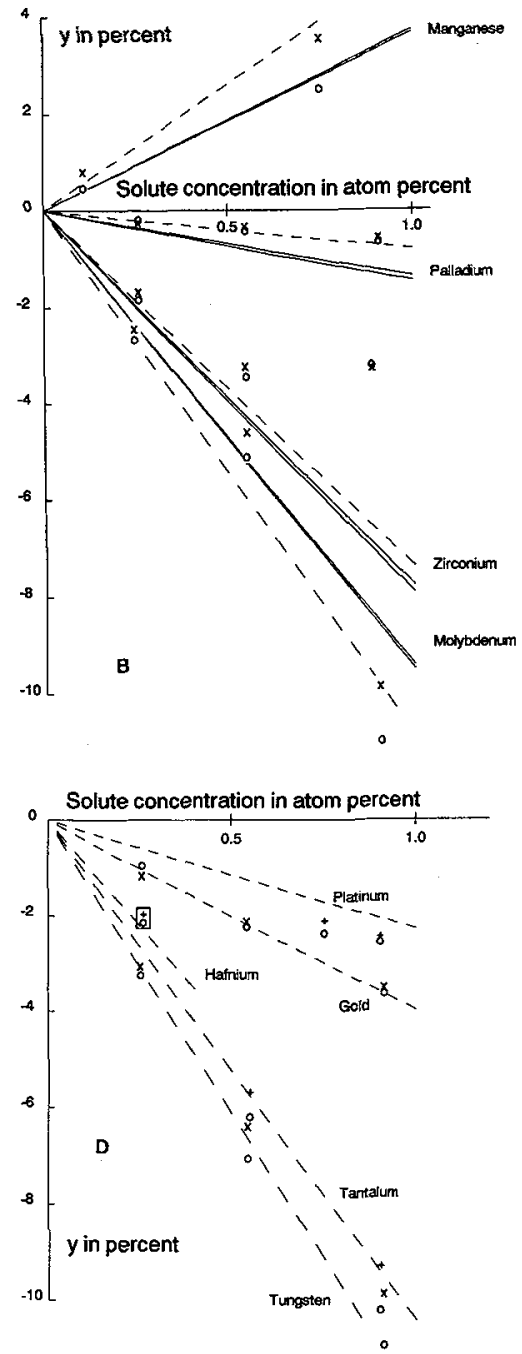

indicates that $\mathrm{Pd}$ has the same moment as $\mathrm{Ni}$ in agreement with the Friedel rule. The alloys with $0.0091 \mathrm{Zr}$ and $0.0055 \mathrm{Zr}$ are not single phase. The alloys with $\mathrm{Mn}$ show a large temperature dependence leading to an overestimation of the low temperature moment. (c) For alloys where the moment decreases rapidly with concentration, precipitation would increase the magnetization. This is most evident in the alloy with $0.0055 \mathrm{~V}$. (d) The theory has not been carried out for the $5 \mathrm{~d}$ solutes. the data points for the single Hf sample are buried under the Ta points in the box. The $\mathrm{Pt}$ moment is less than that of $\mathrm{Ni}$ :

sonvalle, P. and Langlois, P., J. Appl. Phys. 63 (1988) 3043-3045.

[5] Pauthenet, R., J. Appl. Phys. 53 (1982) 8187; 53 (1982) 2029

[6] Jaccarino, V., Walker, L. R. and Wertheim, G. K., Phys. Rev. Lett. 13 (1964) 752. 